\title{
Design of Automatic Braking System Using Interval Type-2 Fuzzy Logic System
}

Dwi Setiady, Oyas Wahyunggoro, Prapto Nugroho

Department of Electrical Engineering and Information Technology

Bulaksumur, Caturtunggal, Kec. Depok, Kabupaten Sleman

Daerah Istimewa Yogyakarta, Yogyakarta, 55281

Indonesia

dwi.setiady@mail.ugm.ac.id,oyas@ugm.ac.id,tatok@ugm.ac.id

\begin{abstract}
The car braking system is a very important part of car safety. The effectiveness of Conventional braking system is strongly influenced by the ability and the driver's experience. It is very vulnerable to accidents if the driver fails to operate the braking system. Because of that, an automation of the braking system is needed that can decrease the accident's impact. The Automatic braking systems require the reasoning to manage information before making a decision that brakes or slows down. There is uncertainty in determining membership function of distance and velocity. It is difficult to determine an exact membership function. In this paper, the system used Interval type-2 fuzzy logic control that can work well in an unstructured environment and have the ability to overcome the uncertainty of information. The result is that the pressure on the brake pads is gradually increased with an average increase of $5.6 \%$. At a distance of $34 \mathrm{~m}$, the pressure is at maximum value.
\end{abstract}

Keywords: Artificial Intelligent System, Automatic Braking System, Type-2 Fuzzy Logic Control, Fuzzy Logic Control

Received: 18 October 2018, Revised 5 January 2019, Accepted 22 January 2019

DOI: $10.6025 / \mathrm{jes} / 2019 / 9 / 2 / 35-43$

\section{(C) 2019 DLINE. All Rights Reserved}

\section{Introduction}

It is undeniable that the current level of traffic accidents is still very high. Based on data compiled by the Indonesian Police, the number of traffic accident in Indonesia for the last quarter tends to increase, which is about 26,000 cases with a death toll of about 7,000 people. Based on these data, about $30 \%$ of these accidents are car accidents [1].

The braking system is one of important thing that supporting the driver's safety. The car's braking system is usually operated manually with the brake pedal. If the driver fails to break, there will be a collision or even accidents that cause damage or victim.

In recent years, a lot of research have been developed for an automatic car braking systems [2][3][4][5]. Some studies rely on mathematical modeling but the fact is that in the case of the braking system, driver's behavior is strongly influenced by the experience, not the appropriate mathematical modeling [2][5]. Because of that, the system needs a model that can apply human reasoning based on experience in a linguistic form that does not need to use complex mathematical models. Fuzzy logic systems are very good at applying human thinking based on experience to handle non-linear systems such as braking systems [2].

Fuzzy was first developed by Lotfi Zadeh and does not yet have the ability to handle uncertainty [6]. In this braking system,

Journal of Electronic Systems Volume 9 Number 2 June 2019 
there is uncertainty information in determining a safe distance at a certain speed when braking. Safe distance is only determined according to the estimations and experiences have been obtained. conventional fuzzy is less effective in dealing with uncertainty [7].

Over the past decade, many researchers have developed Type-2 Fuzzy Logic System (T2FLS) which is an improvement from conventional fuzzy or Type-1 Fuzzy Logic System [8][9][10][11]. Type 2 has a better ability to overcome uncertainty. Compared to T1FLS, T2FLS has more parameters in representing Fuzzy set elements [7]. T2FLS has 3-dimensional functions and an uncertainty range called Footprint-Of-Uncertainty offering additional degrees of freedom to deal with various uncertainties.

Interval Type-2 Fuzzy Set (IT2FLS) is a type of type-2 Fuzzy Set that is widely used because it has lower computational complexity than general T2FS. IT2FLS has easy-to-manage computing that makes IT2FLS easy to implement [12].

This paper will discuss the braking system that applies IT2FLS methods for managing and analyzing the conditions in performing automatic braking. The purpose of this study is to test IT2fls modeling capabilities in handling non-linear systems such as braking systems.

\section{Braking System Design}

\subsection{Car Braking System}

In this paper, the automatic braking system uses IT2FLS modeling to apply brakes when the distance between the car and obstacles is close. The output is the pressure required by the brake pads when clamping the disk plate illustrated in Figure 1.

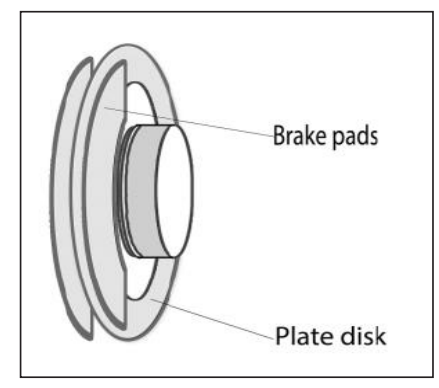

Figure 1. Illustration of the braking system mechanism

It is assumed that the pressure is 0 at minimum and 150 at the maximum. This system will be implemented using the fuzzy type-2 interval Matlab Simulink toolbox [13]. In this car's braking system, there are several parameters that have been ignored, namely engine dynamics, friction, and gravity.

\subsection{System Modeling}

System modeling is generally needed in the development of a system. Figure 2 shows the system process in general. Starting from the input process, the process of fuzzification, up to the results.

\subsubsection{System planning}

The method used is Grid Partition [14]. This partitioning method is often used in designing fuzzy controllers that have few variables as input to controllers. This partitioning strategy requires only a small number of MF for each input. Fuzzy models with 2 inputs and $3 \mathrm{MF}$ in each input will produce 9 Fuzzy if-then rules. Rule modeling uses simple modeling, namely Takagi Sugeno Kang (TSK) model. The rule model is written in Equation (1) [15].

$$
R^{n} \text { : If } \chi_{1} \text { is } \widetilde{X}_{1} \text { and } \tilde{\chi}_{p} \text { then } y \text { is } Y
$$

With $(p=1,2, \ldots, N p)$ is IT2 FS for IT2 FLS. whereas $Y^{n}=\left[y^{n}, \vec{y}^{n}\right]$ is a simple interval. This model is used $y^{n}=y^{n}$.

In Table 1, the attribute is attached. Distance variables have 3 membership functions, namely: near, medium and far. Speed variable which has 3 membership functions, namely: slow, medium, and fast. 


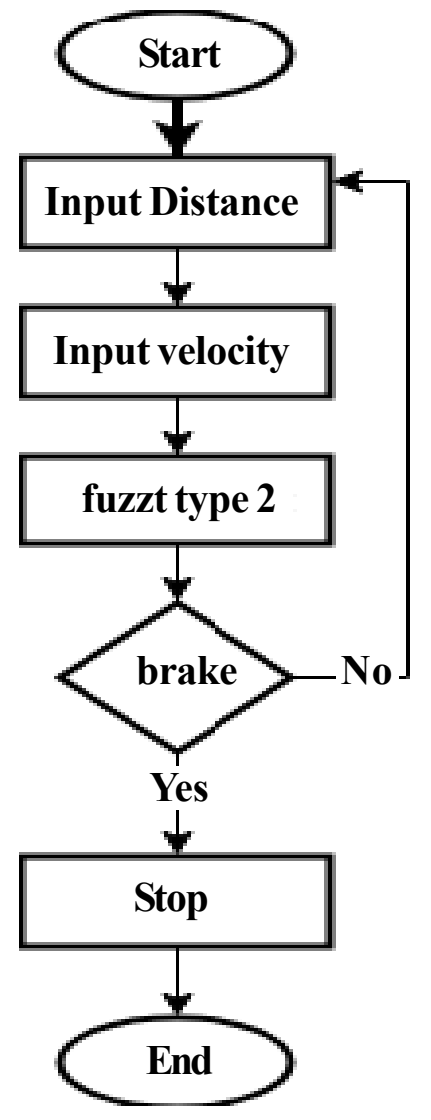

Figure 2. Block diagram Automatic braking system using IT2FLS

\begin{tabular}{|l|c|c|}
\hline Attribute & Unit & Range \\
\hline Distance & Near(1), Medium(2), Far(3) & $0-70(1), 35-105(2), 70-200(3)$ \\
Velocity & Slow(1), Medium(2), Fast(3) & $0-125(1), 35-220(2), 125-250(3)$ \\
\hline
\end{tabular}

Table 1. Data Set Attribute

Design the Membership Function After generating the rule base, the thing to do is to determine the membership function as the basis of fuzzification and defuzzification. The first thing to do is to design a membership function from T1 and become a T1 MF baseline. Furthermore, based on the MF baseline, Footprint of Uncertainty (FOU) was determined to be the presentation of randomness and uncertainty of MF data.

In designing the type 1 membership function using a triangular model and trapezium with each variable has 3 parameters. Distance variable consists of: near, medium, and far. Velocity variables consist of: slow, medium, and fast.

This research using type 1 membership function as the basis for designing the FOU described in the previous stage. Next, determine the two fields of FOU, namely Lower Membership Function (LMF) and Upper Membership Function (UMF). It is assumed that there is no doubt at the center point of type 1 membership function. Illustration of FOU design is shown in Figure 3. 


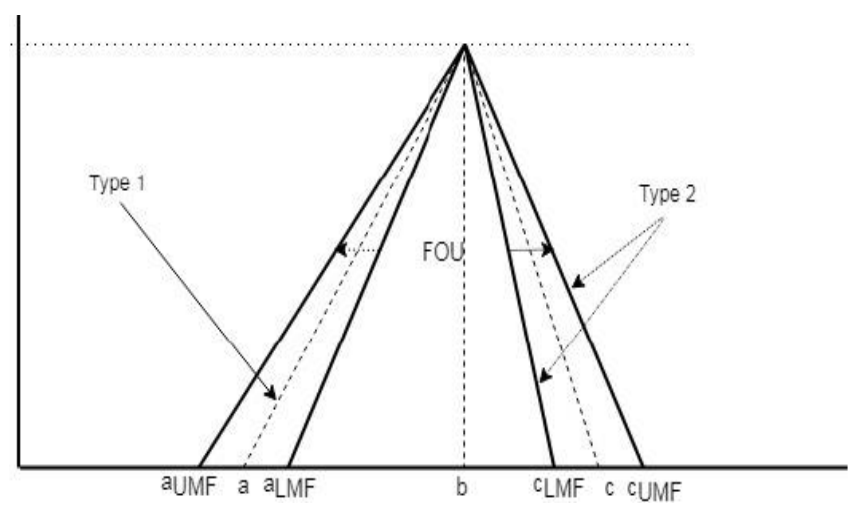

Figure 3. Design of IT2 FOU

$a_{U M F}$ is the left point of the UMF determined by Equation (2), $b a_{U M F}$ is the center point of UMF with Equation (3), $c_{U M F}$ is the right point of UMF with Equation (4), $a_{L M F}$ is the LMF left point with Equation (5), $b c_{L M F}$ is the LMF center point with Equation (6), $c_{L M F}$ is the center point of LMF with Equation (7), $\mathrm{a}$ is the left point T1 MF, $\mathrm{b}$ is the center point $\mathrm{T} 1 \mathrm{MF}$ and $\mathrm{c}$ is the right point $\mathrm{T} 1 \mathrm{MF}$.

The results of the FOU design are shown in Figure 4 and Figure 5.

$$
\begin{aligned}
& a_{U M F}=a-5 \\
& b a_{U M F}=b \\
& c_{U M F}=c+5 \\
& a_{L M F}=a+5 \\
& b c_{L M F}=\mathrm{b} \\
& c_{L M F}=c-5
\end{aligned}
$$

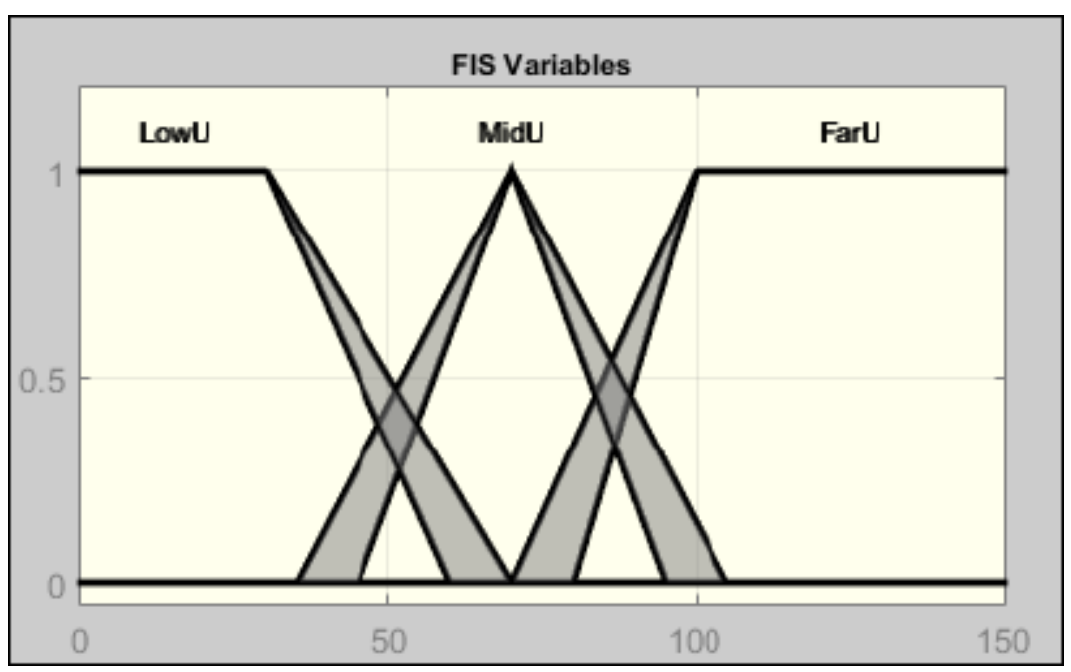

Figure 4. Distance membership functions at IT2FLS 


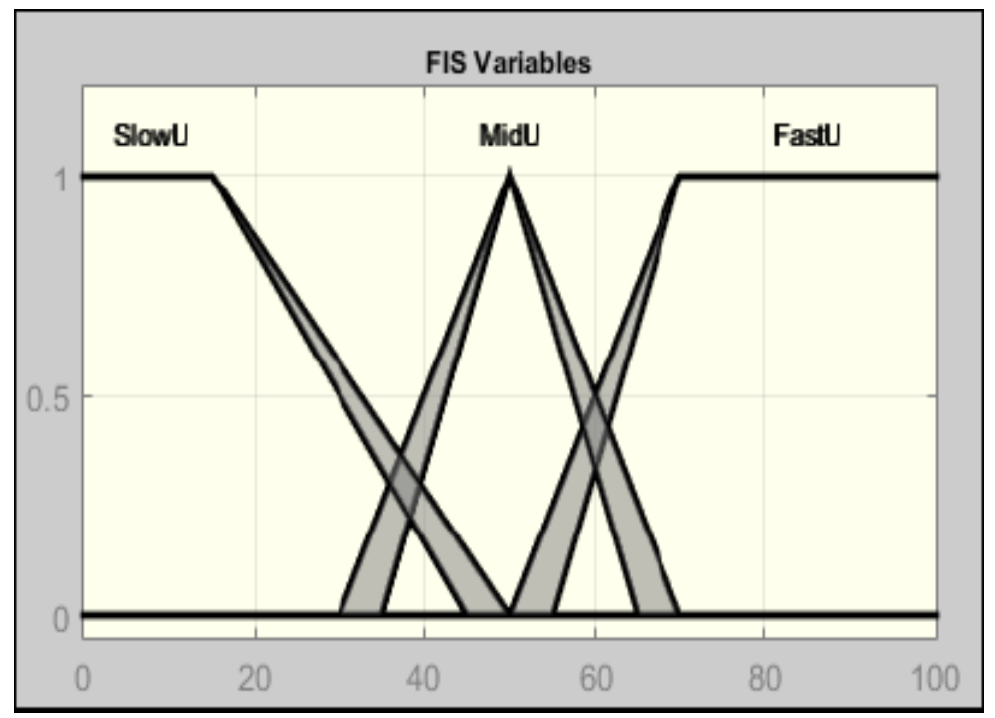

Figure 5. Velocity membership functions at IT2FLS

\section{Design of Interval Type-2 Fuzzy Logic}

In this session, there is an explanation of system design used on automatic braking systems. The system applied is t2fls which will be explained in stages

\subsection{Fuzzyfier}

The input is a vector $\mathrm{n}$ crisp element $x=(x 1, \ldots, X n) \mathrm{T}$ in the discourse universe $X 1 \times X 2 \times \ldots \times X n$ mapped into a type-2 Fuzzy set.

\subsection{Rule Base}

The composition of the rules in set type-2 has no difference with that of type-1 shown in figure 6. The 1-rule for n-input, $x_{1} \in X_{1}$, $\ldots, x_{n} \in X_{n}$, and m-output, $y_{1} \in Y_{1}, \ldots, y_{m} \in Y_{m}$, from type-2 Fuzzy logic systems are given in Equation (8).

$$
R^{p}: I F x_{1} \text { is }{\widetilde{F_{1}}}_{1}^{p} \text { and } x \text { is } \widetilde{F_{n}^{p}}{ }^{p} \text { HEN } \widetilde{Y^{1}} \text { is } p=1,2, \ldots N
$$

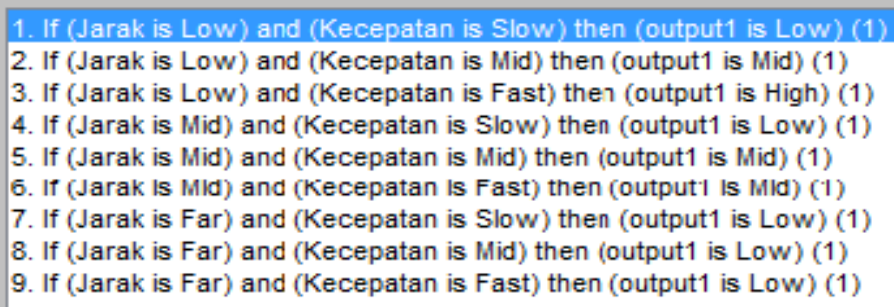

Figure 6. The Base Rule

\subsection{Inference}

The Inference is needed to combine rules and do a mapping from input to output. This is important for calculating the join $\cup$ (union), slices $\cap$ (intersection) the sup-star composition of type 2 relations.

In this FLS, the type-2 Fuzzy set and product t-norm intervals are used, so the input and antecedent results are in the firing set is $\Pi_{n=1}^{p} \mu_{\widetilde{F}_{n}}\left(x_{n} \equiv F^{p}\left(x^{\prime}\right)\right.$ the type 2 interval set, as seen in Equation (9). 


$$
F^{p}\left(x^{\prime}\right)=\left[\underline{f}^{p}\left(x^{\prime}\right), \underline{f}^{-p}\left(x^{\prime}\right)\right] \equiv\left[\underline{f}^{p}, \underline{f}^{-p}\right]
$$

where

$$
\underline{f}^{p}\left(x^{\prime}\right)=\underline{\mu}_{\widetilde{F}_{1}^{1}}\left(x 1^{\prime}\right) \times \ldots \times \underline{\mu}_{\widetilde{F}_{n}} p\left(x n^{\prime}\right)
$$

and

$$
\bar{f}^{p}\left(x^{\prime}\right)=\bar{\mu}_{\widetilde{F}_{1}}\left(x 1^{\prime}\right) \times \ldots \times \bar{\mu}_{\widetilde{F}_{n}^{p}}\left(x n^{\prime}\right)
$$

\subsection{Type Reducer}

The type reducer produces a type 1 Fuzzy set which will then become a defuzzification input to get the crisp value. For this research, center of sets $\left(Y_{c o s}\right)$ type reduction which is used is stated in Equation (12).

$$
Y_{\cos }(x)=\cup f^{n} \in f^{n}\left(x^{\prime}\right) \frac{\sum_{n=1}^{p} f^{n} y^{n}}{\sum_{n=1}^{p} f^{n}}=\left[y_{1}, y_{r}\right]=\left[y^{n} \in Y^{n}\right]
$$

with

$$
y_{l}=\min _{k \in[1, N-1]} \frac{\sum_{n=1}^{k} \bar{f}^{n} \underline{y}^{n}+\sum_{n=k+1}^{N} \underline{f}^{n} \underline{y}^{n}}{\sum_{n=1}^{k} \bar{f}^{n}+\sum_{n=k+1}^{k} \underline{f}^{n}} \equiv \frac{\sum_{n=1}^{L} \bar{f}^{n} \underline{y}^{n}+\sum_{n=L+1}^{k} \underline{f}^{n} \underline{y}^{n}}{\sum_{n=1}^{L} \bar{f}^{n}+\sum_{n=L+1}^{k} \underline{f}^{n}}
$$

and

$$
y_{r}=\max _{k \in[1, N-1]} \frac{\sum_{n=1}^{k} \underline{f}^{n} \bar{y}^{n}+\sum_{n=k+1}^{N} \bar{f}^{n} \bar{y}^{n}}{\sum_{n=1}^{k} \underline{f}^{n}+\sum_{n=k+1}^{N} \bar{f}^{n}} \equiv \frac{\sum_{n=1}^{R} \underline{f}^{n} \bar{y}^{n}+\sum_{n=R+1}^{k} \bar{f}^{n} \bar{y}^{n}}{\sum_{n=1}^{R} \underline{f}^{n}+\sum_{n=R+1}^{N} \bar{f}^{n}}
$$

Where $L$ and $R$ switch points are determined by Equation (15) and (16).

$$
\begin{aligned}
& \underline{y}^{L} \leq y_{l} \leq \underline{y}^{L+1} \\
& \bar{y}^{R} \leq y_{r} \leq \bar{y}^{R+1}
\end{aligned}
$$

$y_{l}$ and $y_{r}$ Where $\mathrm{L}$ and $\mathrm{R}$ switches are determined by Equation (15) and (16) [16] (Karnik-Mendel).

\subsection{Defuzzifier}

After passing the type reducer, $Y_{c o s}$ interval value will be obtained. To defuzzify this value, only the average values of and are $y_{l}$ and $y_{r}$ needed. The equation used is Equation (17).

$$
y(x)=\frac{y_{l}+y_{r}}{2}
$$

\section{Results and Discussion}

This system was implemented using Matlab. Testing was done by setting the initial speed of a car, then place objects at a certain distance right in front of the car. This test was carried out by testing a system that applies to brake using the IT2FLS system. The car speed value was $100 \mathrm{~km} / \mathrm{hr}$. The distance to the object is $100 \mathrm{~m}$. The test illustration is shown in Figure 7.

For the evaluation of the test refers to the formula for a safe distance determined by the National Police Corps, which was 3 
seconds. If the speed of the car was at $100 \mathrm{~km} / \mathrm{h}$, the safety distance to the object or the obstacle in front of it was $83,3 \mathrm{~m}$. Based on the above calculations, braking must started at a distance of $83,3 \mathrm{~m}$ from the obstacle because it had passed the safe distance.

In testing the automatic braking system, the pressure starts at a distance of $100 \mathrm{~m}$ with a pressure value of 4,68 . Pressure increases slowly as the distance of the obstacle approaches. The value of brake pressure has exceeded $50 \%$ at a distance of 80 which is 61,6 shown in table 2.

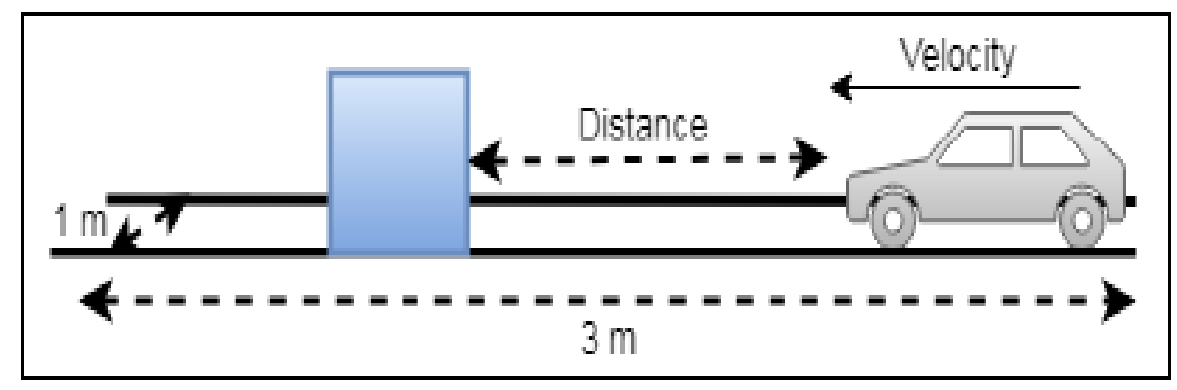

Figure 7. Illustration of Braking System Testing

\begin{tabular}{|c|c|c|}
\hline \multirow{2}{*}{ No } & \multicolumn{2}{|c|}{ Result of Bracking System } \\
\cline { 2 - 3 } & Distance & Preasure of Brake \\
\hline 1 & 34 & 150 \\
\hline 2 & 36 & 148,7 \\
\hline 3 & 40 & 143,3 \\
\hline 4 & 44 & 137,8 \\
\hline 5 & 48 & 125,2 \\
\hline 6 & 52 & 111,4 \\
\hline 7 & 56 & 98,4 \\
\hline 8 & 60 & 86,02 \\
\hline 9 & 64 & 81,18 \\
\hline 10 & 68 & 76,93 \\
\hline 11 & 72 & 72,46 \\
\hline 12 & 76 & 67,18 \\
\hline 13 & 80 & 61,6 \\
\hline 14 & 84 & 46,32 \\
\hline 15 & 88 & 32,4 \\
\hline 16 & 92 & 19,6 \\
\hline 17 & 96 & 9,12 \\
\hline 18 & 100 & 4,68 \\
\hline
\end{tabular}

Table 2. Result of the IT2FLS Bracking System 


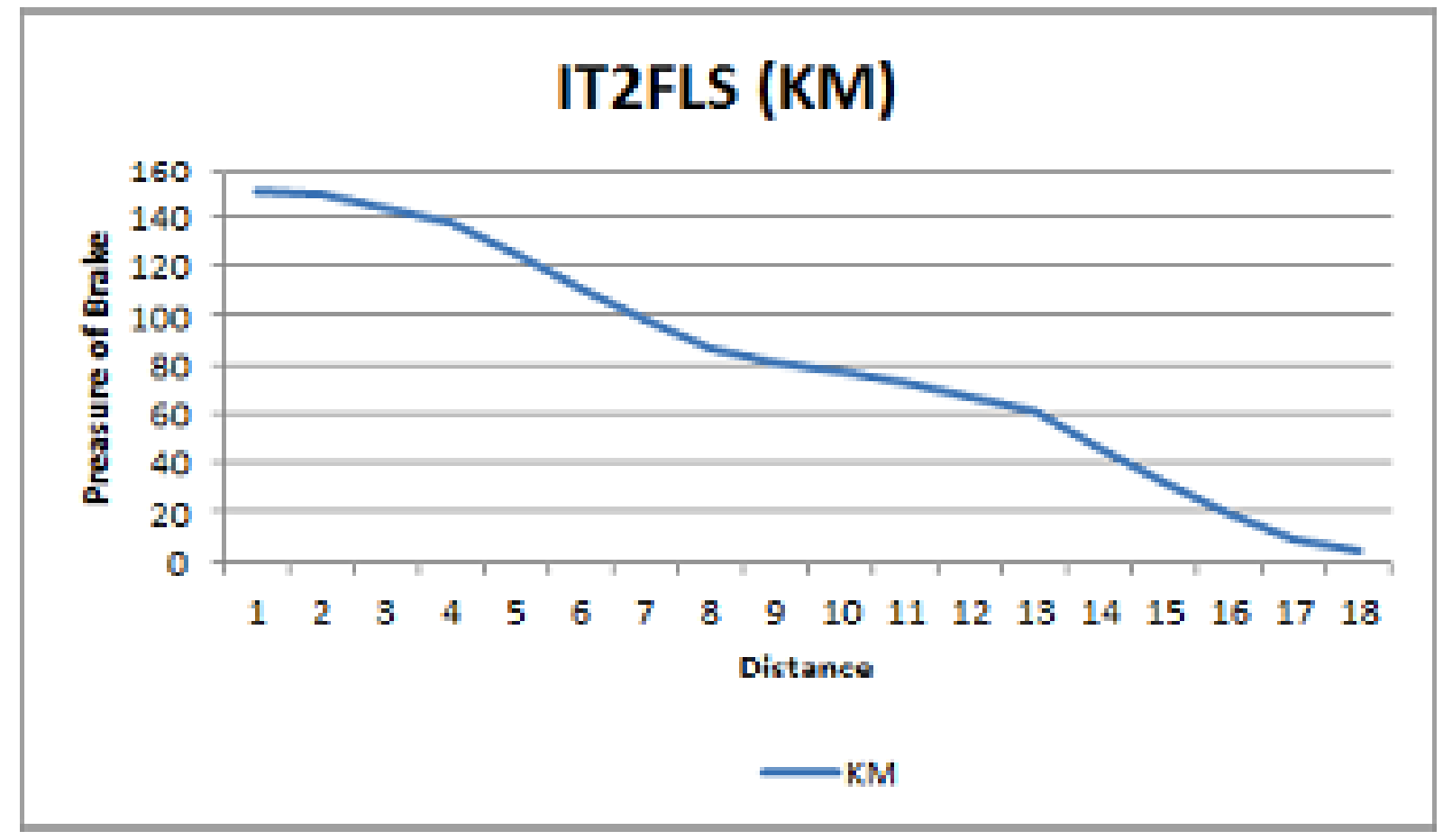

Figure 8. Result test for IT2FLS (KM) for distance

The result of the pressure change on the brake lining is shown in figure 8 . At a distance of $34 \mathrm{~m}$, the pressure on the brake pads is at a maximum value of 150 . The average pressure change produced reaches a maximum value of $5.6 \%$.

\section{Conclusion}

In this paper, we discuss the automatic braking system using IT2FLS which has two inputs and one output. Input in the form of distance and speed while the output is in the form of pressure on the brake pads. The results show an increased pressure on the brake pads smoothly with an average value of $5.6 \%$. This system reaches pressure on the canvas with a maximum value of $34 \mathrm{~m}$. For further research, it can be improved by adding input and adding rules so that the system's capabilities are better.

\section{References}

[1] Korlantas-irsms.info. (2018). KORLANTAS POLRI - Accident Reports by Polda. [online] Available at: http://www.korlantas irsms.info/graph/accidentTypeTable [Accessed 1 Nov. 2018].

[2] Hirulkar, S., Damle, M., Rathee, V., Hardas, B. (2014). Design of Automatic Car Breaking System Using Fuzzy Logic and PID Controller. 2014 International Conference on Electronic Systems, Signal Processing, and Computing Technologies.

[3] Jin, P., Kim, J., Kim, J. (2015). Design of unmanned vehicle advanced braking system using smart motor. $201512^{\text {th }}$ International Conference on Ubiquitous Robots and Ambient Intelligence (URAI).

[4] Chen, X., Zhang, J., Liu, Y. (2016). Research on the Intelligent Control and Simulation of Automobile Cruise System Based on Fuzzy System. Mathematical Problems in Engineering, 2016, p 1-12.

[5] Rizianiza, I., Djafar, A. (2017). Design car braking system using Mamdani Fuzzy Logic Control. $20174^{\text {th }}$ International Conference on Electric Vehicular Technology (ICEVT).

[6] Zadeh, L. (1975). The concept of a linguistic variable and its application to approximate reasoning-I. Information Sciences, $8(3), 199-249$. 
[7] Naik, K., Gupta, C. (2017). Performance comparison of Type-1 and Type-2 fuzzy logic systems. In: $20174^{\text {th }}$ International Conference on Signal Processing, Computing and Control (ISPCC).

[8] Castillo, O. (2012). Type-2 Fuzzy Logic in Intelligent Control Applications. Berlin, Heidelberg: Springer Berlin Heidelberg.

[9] Karnik, N., Mendel, J. (n.d.). Introduction to type-2 fuzzy logic systems. 1998 IEEE International Conference on Fuzzy Systems, In: Proceedings. IEEE World Congress on Computational Intelligence (Cat. No.98CH36228).

[10] Castillo, O. , Melin, P. (2012). Recent advances in interval type-2 fuzzy systems. Heidelberg: Springer.

[11] Saxena, V., Yadala, N., Chourasia, R. Rhee, F. (2017). Type reduction techniques for two-dimensional interval type-2 fuzzy sets. In: 2017 IEEE International Conference on Fuzzy Systems (FUZZ-IEEE).

[12] Wu, D., Mendel, J. M. (2014). Designing practical interval type-2 fuzzy logic systems made simple, In: 2014 IEEE International Conference on Fuzzy Systems (FUZZ-IEEE), 2014.

[13] Taskin, A., Kumbasar, T. (2015). An Open Source Matlab/Simulink Toolbox for Interval Type-2 Fuzzy Logic Systems. In: 2015 IEEE Symposium Series on Computational Intelligence.

[14] Castillo, O., Melin, P., Kacprzyk, J., Pedrycz, W. (2007). Type-2 Fuzzy Logic: Theory and Applications. In: 2007 IEEE International Conference on Granular Computing (GRC 2007).

[15] Sajiah, A., Setiawan, N., Wahyunggoro, O. (2016). Interval type-2 fuzzy logic system for diagnosis coronary artery disease. Communications in Science and Technology, 1(2) 1-55.

[16] Wu, D. (2013). A Brief Tutorial on Interval Type-2 Fuzzy Sets and Systems. p.1-13. 